\title{
The Effect of Electrode Arrangements on Concrete Defect Test Using Electrical Resistance Tomography
}

\author{
Xiaojun Zhu ${ }^{1, a}$, Lei Qin ${ }^{1, b, *}$, Hongwei Ren ${ }^{2, c}$, Biqin Dong ${ }^{3, d}$, Xing Feng ${ }^{3, e}$ \\ ${ }^{1}$ School of Civil Engineering and Architecture, University of Jinan, Jinan 250022, China. \\ ${ }^{2}$ School of electrical engineering, University of Jinan, Jinan 250022, China \\ ${ }^{3}$ School of Civil Engineering, Guangdong Province Key Laboratory of Durability for Marine Civil \\ Engineering, Shenzhen University, Shenzhen 518060, China \\ azhuxiaojun9969@163.com, bcea_qinl@ujn.edu.cn, ccse_renhw@ujn.edu.cn, dxingf@szu.edu.cn, \\ eincise@szu.edu.cn
}

Keywords: Electrical Resistance Tomography; Electrode Arrangements; Imaging Reconstruction.

\begin{abstract}
Electrical resistance tomography (ERT) is an imaging modality in which the internal conductivity distribution of the target is reconstructed on the basis of surface voltage measurements. It is assumed that the spatial resolution of the reconstructed images with an electrical resistance tomography system can be consummated by electrode arrangements. In this thesis, the key issues with electrode arrangements will be discussed. To investigate the effect of different electrode arrangements, a series of simulation and experiments were designed. Compared to uniform distribution of electrode arrangements, simulation and experimental results show that the imaging reconstruction is varied. Distribution uniformity of electrodes is directly related to the quality of the imaging.
\end{abstract}

\section{Introduction}

ERT is a class of diffuse tomography modalities which intent to estimate the interior distribution from boundary measurements [1, 2, 3]. Owning to the advantages of high speed, safety, non-invasiveness and low-cost, Electrical resistance tomography (ERT) is attracting more and more researches studying it to apply in structural health monitoring. As we all know, concrete is a stone-like construction material mainly consisted of cement, coarse and fine aggregate, water and some concrete additive. Poor conductivity of these compositions lead to high resistance of concrete. Therefore, the application of ERT for non destructive testing of concrete is still in preliminary stage. In 2009, Hou and Lynch [4] performed cracking of fiber-reinforced cementitious composites tests during tensile loading,which demonstrated that ERT can be as a powerful new nondestructive examination tool for health monitoring of cementitious structures. In 2010, the feasibility evaluations of ERT for imaging of the concrete was studied by Karhunen et al. [5,6] The results indicated that ERT could be a feasible technology for non-destructive evaluation of concrete. Crack and reinforcing bar sizes and their respective positions can be reliable estimated. In 2015, Hallaji [7]applied ERT to monitoring damage and unsaturated moisture flow in concrete. Crack and moisture distributions can be well characterized in cementitious materials.

In these studies, all of the concrete specimens were cylindrical and electrodes are well-distributed around the concrete specimens. However, the electrodes can't be completely spaced along the field boundary. Different electrode arrangements can effect imaging quality. But few papers of ERT for effect of electrode arrangements have been reported. In this paper, the effect of electrode arrangements will be discussed.

\section{Experiments}

In order to investigate the effect of electrode arrangements on concrete defect test using electrical resistance tomography, simulation and experiment approaches are adopted at the same time. Based on 
PC's measurement and automation platform, ERT system which adopted PXI platform of NI company is developed by the electric imaging group of University of Jinan. As is shown in fig. 1.

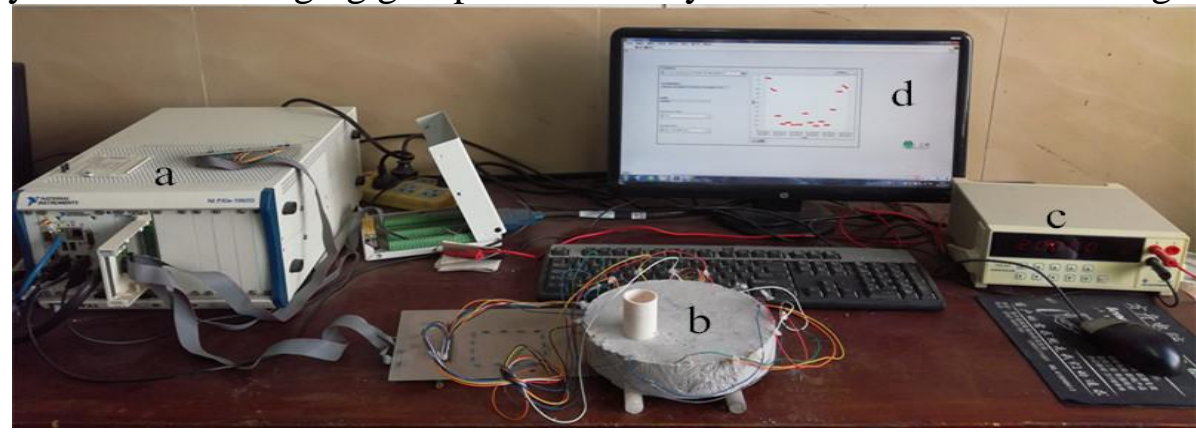

Fig. 1. ERT system. a) PXI Platform. b) Concrete specimen.

c) signal generator. d) Image reconstruction unit.

Four specimens were cast $(20 \mathrm{~cm}$ in diameter, $5 \mathrm{~cm}$ in height) using different electrode arrangements. As is shown in Table1a, fourteen electrodes were placed equidistantly on one side of the specimen, and the remaining two electrodes uniformly surround on another side. As is shown in Table 1b, twelve electrodes were placed equidistantly on one side of the specimen; the remaining four electrodes were placed equidistantly on another side. As is shown in Table 1c, ten electrodes were placed equidistantly on one side of the specimen; the remaining six electrodes were placed equidistantly on another side. As is shown in Table 1d, sixteen electrodes were placed equidistantly around the concrete samples. All the specimens vertically oriented PVC pipe (diameter $2 \mathrm{~cm}$, length $5 \mathrm{~cm}$ ) which simulated the damage was cast in the concrete sample respectively. The PVC pipe was aligned with $\mathrm{y}$-axis, and altitude of the PVC pipe central axis was $-5 \mathrm{~cm}$, that is, horizontally PVC pipe was located in the center of the concrete cylinder.

The mortar specimens had the following mixture proportion: ISO standard sand (67\%), Portland cement $(33 \%)$ by mass. The w/c ration is 0.6 . The specimens should be sealed airtight in plastic after casting. The specimens were removed from their plastic seals and measured after three days .

Table 1 Four cylindrical specimens

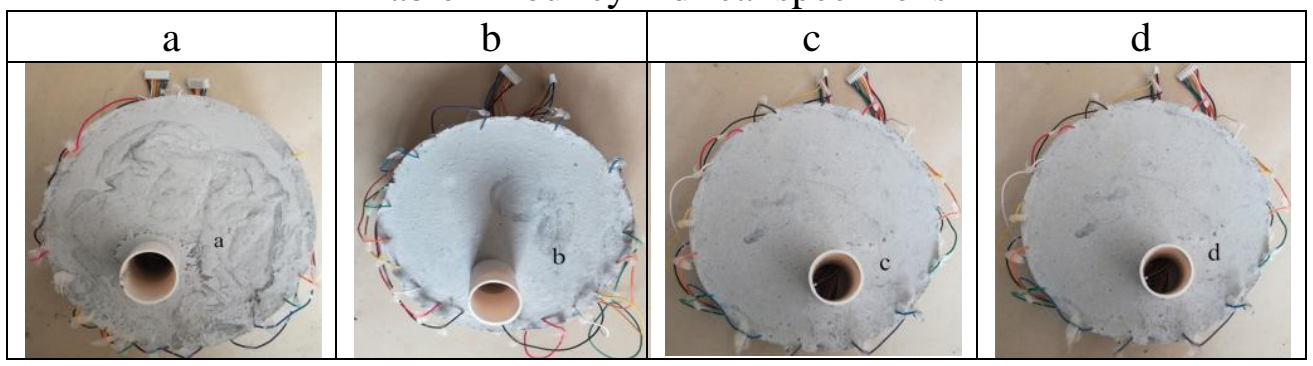

To facilitate the electrical connection between the specimens and ERT equipment, Steel electrode were used, which were buried in the specimens. The steel electrodes were rectangular shape (11 mm long and $5 \mathrm{~mm}$ wide) and were made of thin conductive copper foil. One of the electrodes is shown in fig. 2.

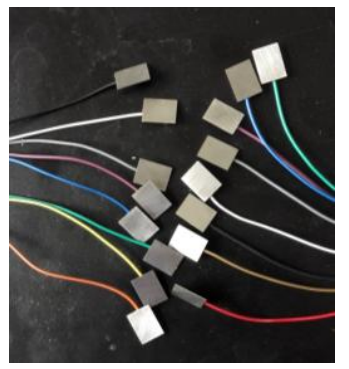

Fig. 2. steel electrode

In this paper, we applied 4-point probe method to calculate the specimen conductivity. A rectangular specimen (dimensions: $40 \mathrm{~mm} \times 40 \mathrm{~mm} \times 160 \mathrm{~mm}$ ) were cast in the same mix proportion. 
In the 4-point probe method, four electrodes uniformly spaced (by a) were cast in the cementitious specimen.As is shown in fig. 3.

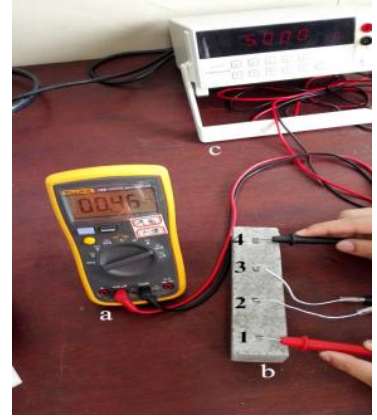

a) digital multimeter b) rectangle block, c) signal generator.1,2,3 and 4 denotes electrodes

(40mm long and $3 \mathrm{~mm}$ wide)

Fig. 3. Measuring specimen conductivity.

For the 4-point probe method, four electrodes uniformly spaced (by a) were cast in the cementitious specimen. The $5 \mathrm{~V}$ voltage was injected via 2 and 3 electrode. The current were measured through 1 and 4 electrode. If the specimen is assumed to be an infinite half-space, then conductivity can be calculated [4]:

$$
\sigma=\frac{I}{V} \cdot \frac{1}{2 \pi a}
$$

According to Eq.1, the specimen conductivity can be calculated. And we proposed the mean value to obtain the accurate conductivity. The conductivity is about $0.025 \mathrm{~S} / \mathrm{M}$. Void damage resistivity approach infinity, thus the reciprocal of resistivity is infinitesimal. To facilitate the calculation, we assume that the conductivity of void damage is about $0.025 \mathrm{~S} / \mathrm{M}$.

\section{Results and discussion}

Through ERT imaging reconstruction, the results of different electrode arrangements simulation and experiments are shown in table 2 .

Table 2. Four sets of specimens with electrode arrangements simulation and experiments

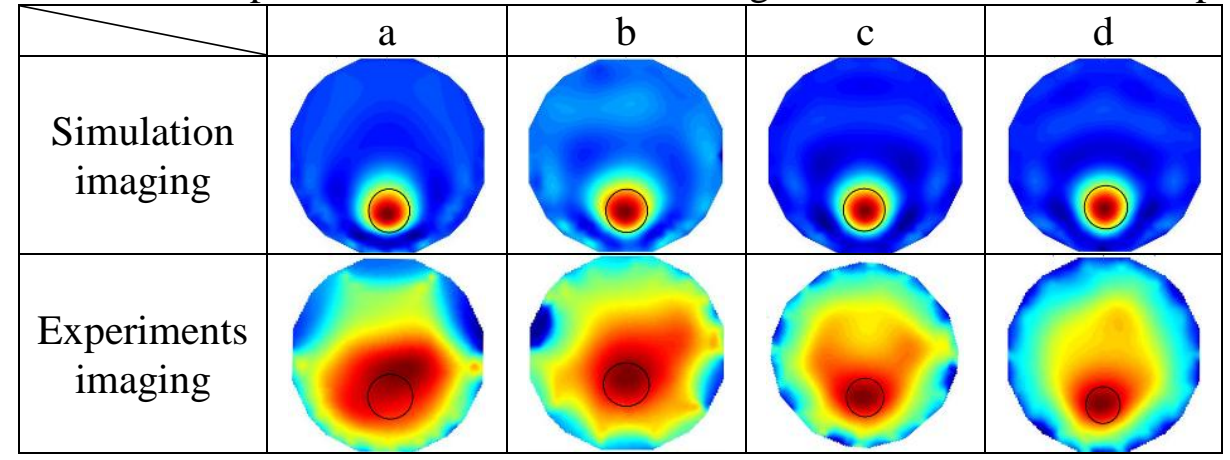

Owing to difference in conductivity between the damage and the matrix, obvious areas of relative high resistance and low resistance can be found. In other words, distinct color change are caused by different conductivity in concrete specimen. From the simulation tests, this phenomenon is very obvious. However, this phenomenon is not obvious in actual tests. This is because that the surface moisture is vulnerable to lose, which makes the interior resistivity distinct. In this paper, to evaluate the quality of the reconstructed imaging, the imagimg correlation coefficient is applied. The correlation coefficient is used to determine the correlation between the simulation imaging and the experiments imaging. From the fig.4, 16 electrodes were placed equidistantly around the concrete samples, the imagimg correlation coefficient is the best in fours concrete specimens, followed a specmen, b specimen, c specimen. From the table 2, compared simulation imaging and experiments imaging, increasing distribution uniformity of electrodes contributes to the localization accuracy of the damage in concrete specimens. Based on the above analysis, the electrodes in the conctrete specimens should be arranged evenly. 


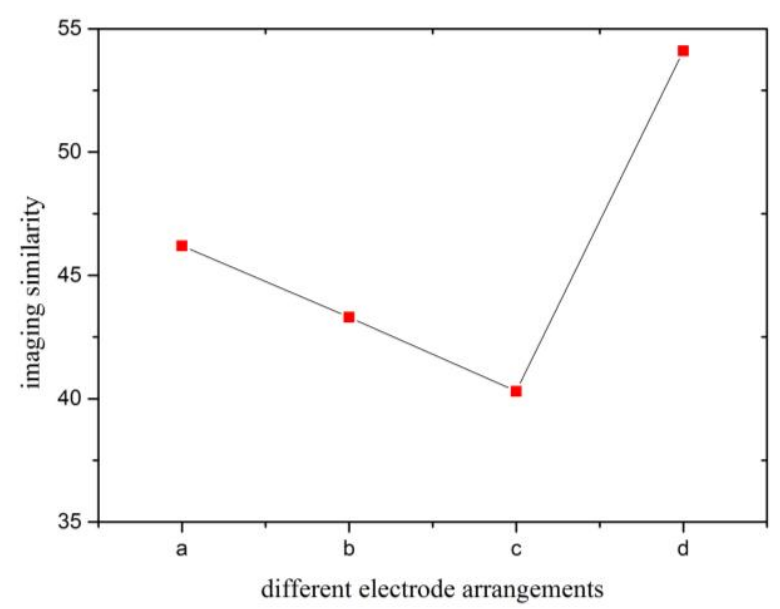

Fig. 4. The relationship different electrode arrangements and imaging similarity

\section{Conclusion}

In this thesis, we adopt simulation and experiment to investigate the effect of electrode arrangements on concrete defect test using electrical resistance tomography. The results showed that the resolution of the ERT imaging in the damage localization was enhanced with the increase of distribution uniformity of electrodes obviously.The reason for distribution uniformity of electrodes increasing is that uniformity of electric field line in the concrete increases. Thus, the electrodes should be arranged uniformly to obtain better imaging quality.

\section{Acknowledgements}

The financial support is from the National Natural Science Foundation of China (51378239).

\section{References}

[1] York T. Status of electrical tomography in industrial applications [J]. Journal of Electronic Imaging, 2001,10(3):608-19.

[2] Tapp H, Peyton A, Kemsley E, et al. Chemical engineering applications of electrical process tomography[J]. Sensors and Actuators B: Chemical, 2003, 92(1):17-24

[3] Zhang L, Xu G, Xue Q, et al. An iterative thresholding algorithm for theinverse problem of electrical resistance tomography [J]. Flow Measurement \& Instrumentation, 2013, 33 (10): 244 -250 .

[4] Hou T C, Lynch J P. Electrical Impedance Tomographic Methods for Sensing Strain Fields and Crack Damage in Cementitious Structures [J]. Journal of Intelligent Material Systems \& Structures, 2009, 20(11):1363-1379.

[5] Karhunen K, Seppänen A, Lehikoinen A, et al. Electrical resistancetomography approach for localizing reinforcing bars in concrete[M]//Concrete Under Severe Conditions, Two Volume Set. 2010.

[6] Karhunen K, Seppänen A, Lehikoinen A, et al. Electrical ResistanceTomography imaging of concrete[J]. Cement \& Concrete Research, 2010, 40(1):137-145.

[7] Hallaji M. Monitoring Damage and Unsaturated Moisture Flow in Concrete with Electrical Resistance Tomography (ERT)[J].Dissertations \& Theses - Gradworks, 2015. 